\title{
Razonamiento Estadístico en Estudiantes de Ingeniería
}

Stella Maris Figueroa

y Maria Andrea Aznar

\begin{abstract}
Resumen
En este trabajo se presenta un análisis de las resoluciones de 140 estudiantes de Estadística de la Facultad de Ingeniería de la Universidad Nacional de Mar del Plata. Las producciones surgen de la comparación de distribuciones de datos que requiere relacionar medidas de tendencia central y de variabilidad en forma gráfica y analítica. Los resultados obtenidos fueron estudiados en el marco del Enfoque Ontosemiótico del Conocimiento y la Instrucción Matemática, identificando las prácticas matemáticas utilizadas con algunas habilidades definidas, en el modelo de Gal, como componentes del razonamiento estadístico. La mayoría de los estudiantes efectuó un análisis sobre la ubicación de la media en la distribución de los datos, según la simetría y variabilidad, pero encontró dificultades para evidenciar las habilidades del conocimiento estadístico pretendidas en el estudio. Casi el total del resto de los estudiantes, resolvió correctamente el problema, traduciendo las gráficas a tablas de frecuencias, a pesar de la información incompleta de los gráficos. Los resultados obtenidos alertan sobre aspectos a considerar en propuestas didácticas que integren el análisis gráfico y analítico de los datos para el desarrollo de habilidades acordes al razonamiento estadístico requerido en estudiantes de ingeniería.
\end{abstract}

Palabras clave: Razonamiento estadístico, Habilidades, Gráficos estadísticos, Enfoque Ontosemiótico. 


\title{
Statistical Reasoning in Engineering Students
}

\begin{abstract}
This work presents an analysis of the resolutions of 140 students of Statistics from the Faculty of Engineering of the Universidad Nacional de Mar del Plata. The productions arise from the comparison of data distributions that requires relating measures of central tendency and variability in a graphical and analytical way. The results obtained were studied within the framework of the Ontosemiotic Approach to Knowledge and Mathematical Instruction, identifying the mathematical practices used with some defined skills, in the Gal's model, as components of statistical reasoning. Most of the students carried out an analysis on the location of the mean in the distribution of the data, according to symmetry and variability, but found difficulties in evidencing the statistical knowledge skills sought in the study. Almost the total of the rest of the students correctly solved the problem, translating the graphs into frequency tables, despite the incomplete information in the graphs. The obtained results warn about aspects to consider in didactic proposals that integrate the graphic and analytical analysis of the data for the development of skills according to the statistical reasoning required in engineering students.
\end{abstract}

Keywords: Statistical reasoning, Skills, Statistical graphics, Ontosemiotic Approach.

Nombre del trabajo: Razonamiento Estadístico en Estudiantes de Ingeniería Nombre y apellido de los autores: Stella Maris Figueroa y Maria Andrea Aznar Integrantes del Grupo de Investigación en Enseñanza de la Matemática en Carreras de Ingeniería. Dirección institucional: Juan B. Justo 2002. 1er piso.

Lugar de trabajo: Facultad de Ingeniería. Anexo.

Tel. edificio central: 0223-481-6600. Universidad Nacional de Mar del Plata.

Tel. Stella Figueroa: 223-5769090

Dirección electrónica: stellafigueroa@fi.mdp.edu.ar; maznar@fi.mdp.edu.ar 


\section{Introducción}

El acelerado avance tecnológico con el uso de plataformas que posibilitan un mayor aprovechamiento de datos generados por las empresas, ha llegado a replantearnos la manera de comprender el mundo, y en particular, la comprensión de los datos en esta época de la sociedad del conocimiento. Si se plantean preguntas adecuadas a los datos, se puede lograr una visión global del problema en la empresa y en los comportamientos de sus clientes. No sólo a las grandes empresas puede resultarles de interés el análisis de datos ya que, en contextos de incertidumbre, el planteo de preguntas y la comprensión de sus respuestas contribuyen a la toma de decisiones y valoración de riesgos.

En este escenario, la formación del ingeniero en la toma de decisiones que surge del análisis de datos, requiere indefectiblemente, acompañar estos cambios. La enseñanza de la estadística en carreras de ingeniería, pretende que el estudiante se involucre en la resolución de un problema que requiere del análisis de datos y de esta manera, pueda darle sentido a los objetos estadísticos intervinientes en su aprendizaje. Al respecto, Batanero (2013) define el sentido estadístico con tres componentes: la comprensión de las ideas estadísticas fundamentales que contribuyeron al desarrollo de la estadística y son necesarias en la resolución de problemas estadísticos, la competencia en el análisis de datos, y el razonamiento para realizar inferencias o tomar decisiones a partir de los datos. El desarrollo del sentido estadístico en estudiantes de ingeniería requiere considerar la Estadística como la ciencia de los datos, donde los números no están vacíos de contenido sino que son datos, presentes en el contexto del problema, que adquieren significados. En consecuencia, el cálculo de medidas descriptivas debe ir acompañado de su significado, es decir, debe ir asociado a la interpretación de cada una de esas medidas en el contexto en el que se definen los datos. Sin embargo, en la mayoría de los textos de estadística de nivel universitario, no se da un espacio de análisis a los significados de las medidas calculadas y estos significados no son construidos por el estudiante de manera espontánea. En el aula se observa en repetidas ocasiones, que el estudiante de ingeniería da como respuesta la definición de una medida, cuando se le está preguntando por su significado. Tampoco relaciona la forma y la variabilidad de la distribución de los datos con las medidas de tendencia central que pueden resumirlos, ni relacionan la variable aleatoria con la variable estadística del problema. Entonces surge preguntarse cuáles habilidades son necesarias desarrollar en el estudiante de ingeniería para el tratamiento de estas dificultades.

Una primera respuesta a esta pregunta la proporcionan Batanero, Godino, Green, Holmes y Vallecillos (1994) y Curcio (1989) al resaltar la necesidad de que los estudiantes adquieran habilidades en la lectura crítica de los datos. Entre dichas habilidades se destaca la interpretación de los gráficos estadísticos. Como señalan (Arteaga, Batanero, Díaz y Contreras, 2009), el lenguaje gráfico tiene un papel esencial en la organización, descripción y análisis de datos donde la capacidad de obtener una nueva información, al cambiar de un sistema de representación a otro, se constituye en una de las formas básicas del razonamiento estadístico. En este trabajo se expondrá un análisis de producciones de estudiantes de Ingeniería, en tareas planteadas para evaluar la vinculación entre los parámetros de distintas poblaciones, con sus representaciones gráficas respectivas, a través de la interpretación global de los datos.

El análisis está enmarcado en constructos teóricos que se exponen a continuación.

\section{Marco Teórico \\ 2.1 Enseñanza de la Estadística en Ingeniería}

Para el estudio sobre los proyectos de mejora de la enseñanza de la Ingeniería, el Consejo Federal de Decanos de Ingeniería (CONFEDI) selecciona un Mo- 
delo Educativo que marca un cambio de enfoque al considerar una enseñanza por competencias. En ese contexto, definió su Primer Acuerdo sobre Competencias Genéricas, en mayo de 2007, señalando que:

Competencia es la capacidad de articular eficazmente un conjunto de esquemas (estructuras mentales) y valores, permitiendo movilizar (poner a disposición) distintos saberes, en un determinado contexto con el fin de resolver situaciones profesionales.

Esta definición establece que las competencias aluden a capacidades complejas e integradas, están relacionadas con saberes (teórico, contextual y procedimental), se vinculan con el saber hacer (formalizado, empírico, relacional), están expresadas en el contexto profesional (entendido como la situación en que el profesional debe desempeñarse o ejercer), referidas al desempeño profesional que se pretende (entendido como la manera en que actúa un profesional técnicamente competente y socialmente comprometido) y permiten incorporar la ética y los valores.

Las competencias genéricas y específicas por carrera, que consideran las características mencionadas, se incluyen en la Propuesta de Estándares de Segunda Generación para la Acreditación de Carreras de Ingeniería en la República Argentina. (Libro Rojo de CONFEDI, 2018).

En este contexto, el cambio de paradigma hacia una enseñanza por competencias, considera una formación centrada en el estudiante de ingeniería, integral, pertinente, propositiva y contextualizada, con apoyo de las tecnologías. Por un lado, se pone el énfasis en el desarrollo de habilidades de metacognición para el autoaprendizaje continuo, junto a las habilidades de comunicación, para el trabajo en equipo y el uso de la tecnología. Por otro lado, se pretende una formación que integre los saberes «conocer», «hacer»y «ser», es decir, que integre los conocimientos teóricos, los procedimientos involucrados y los valores que subyacen en toda formación, para la resolución de problemas de la ingeniería que den respuestas de calidad a las grandes demandas del desarrollo sostenible y sustentable del estado, el país y el mundo.

Para acompañar estos cambios, la formación del ingeniero en estadística requiere de la resolución de problemas de la ingeniería que involucre el análisis de datos y la toma de decisiones, para que de esta forma, el futuro ingeniero adquiera un alto nivel de dominio en la competencia genérica de resolución de problemas, dando sentido a los objetos estadísticos intervinientes en su aprendizaje.

Al respecto, en la Facultad de Ingeniería de la Universidad Nacional de Mar del Plata, el espacio curricular Estadística Básica, está organizado como un todo, con una doble estructura: por un lado, la resolución de problemas, que introduce al estudiante en el análisis de datos y en la alfabetización y el razonamiento estadísticos, y por el otro, la teoría de probabilidades, cuyas variables aleatorias asociadas a las variables estadísticas de los problemas de análisis de datos, son el puente que unen y fundamentan esta organización de los contenidos, como se muestra en la figura 1.

Esta propuesta de mejora fue presentada en la XXXI Reunión Latinoamericana de Matemática Educativa, pág. 257. (S. Figueroa y S. Baccelli, 2018).

La reorganización de contenidos contribuyó a definir los resultados de aprendizaje de la asignatura, entendidos como las competencias de la asignatura, que por pertenecer a las ciencias básicas de la ingeniería, están alineados principalmente con las competencias genéricas y con parte de algunas competencias específicas de la ingeniería industrial, por ser la carrera que mayor cantidad de contenidos estadísticos precisa, referidos al control de calidad y a la toma de decisiones respecto del análisis estadístico de procesos. 


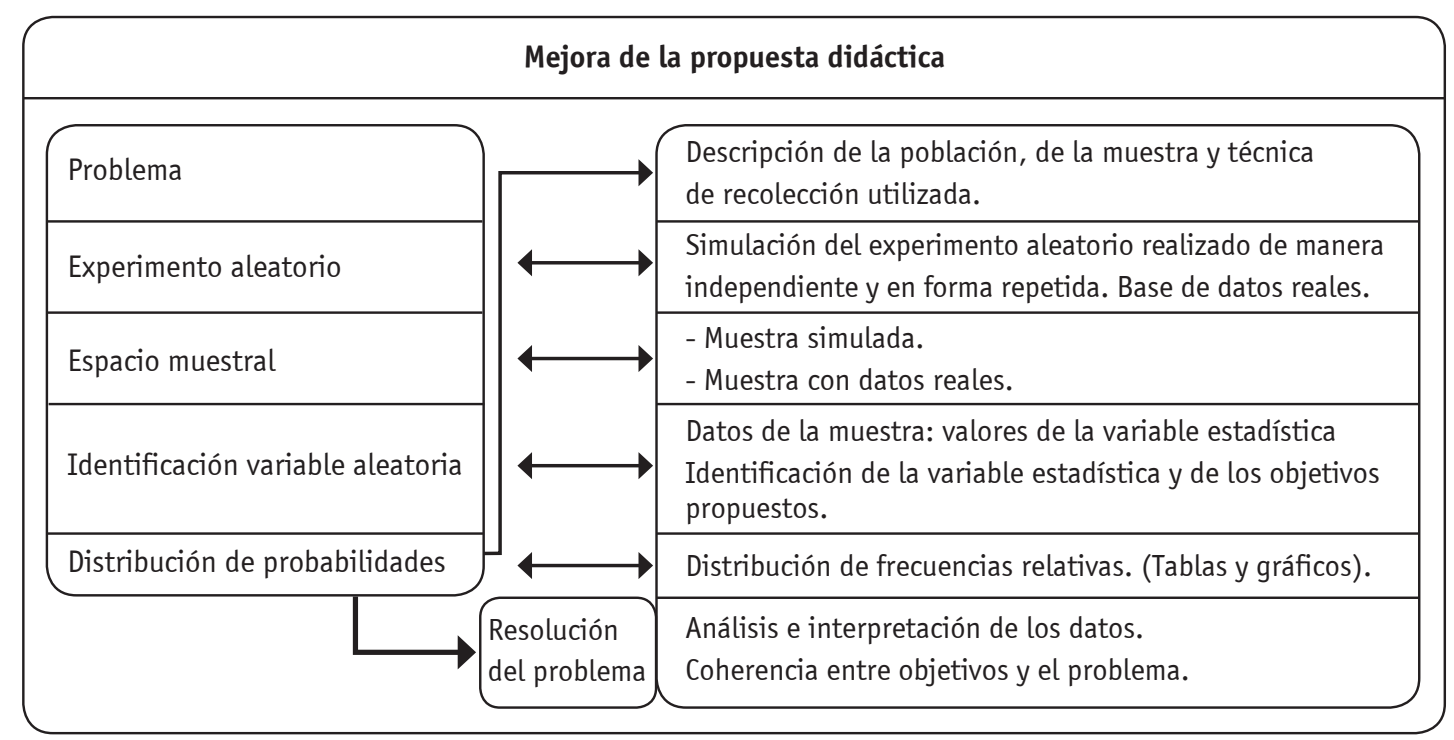

Figura 1.

Propuesta de Enseñanza de Estadística Básica a partir de la resolución de problemas de análisis de datos.

\subsection{Alfabetización, Razonamiento y Pensamiento Estadístico}

Dentro de la Educación Estadística hay conceptos primitivos de los que Tauber (2010), plantea la necesidad de su clarificación y consistencia. Estos conceptos refieren a los procesos cognitivos de alfabetización, razonamiento y pensamiento estadístico. En esta clasificación propuesta por Tauber (2010), Ben-Zvi y Garfield (2004), los autores describen habilidades que caracterizan estos procesos que resumen las ideas de mayor aceptación entre los educadores estadísticos e investigadores sobre el tema, a las cuales este trabajo adhiere:

- Alfabetización estadística: incluye las habilidades básicas que se utilizan para realizar una lectura e interpretación básica de la información y de los resultados presentados en reportes periodísticos 0 investigaciones. Estas habilidades se refieren a organizar datos, construir y presentar tablas y trabajar con distintas representaciones de datos. También incluye una comprensión básica de conceptos, vocabulario y símbolos, y de la probabilidad como una medida de la incertidumbre.

- Razonamiento estadístico: se refiere al cómo razonar y dar sentido a la información estadística. Involucra hacer interpretaciones basadas en un conjunto de datos, representar o resumir datos. También comprende las relaciones entre conceptos, o combinar ideas sobre los datos y las posibilidades. Razonar, en este sentido, significa comprender y ser capaz de explicar procesos estadísticos y de interpretar, de manera global, los resultados estadísticos.

- Pensamiento estadístico: Involucra la comprensión del por qué y cómo se realizan las investigaciones y el papel de las «grandes ideas estocásticas» implícitas en ellas. Estas ideas incluyen la naturaleza de la variación, cuándo y cómo usar los métodos más apropiados del análisis de datos, tales como resúmenes numéricos y gráficos. En este pensamiento interviene una comprensión de la naturaleza del muestreo, cómo hacer inferencias a la población y cómo diseñar experimentos con el objetivo de establecer causas. Además comprende cómo usar los modelos para simular fenómenos aleatorios y por qué sirven para estimar probabilidades. Este pensamiento también implica utilizar el contexto de 
un problema de investigación y dar conclusiones, reconocer y comprender los procesos completos (desde proponer preguntas a recolectar los datos para elegir el análisis y la prueba de hipótesis que corresponda). Por último, el pensamiento estadístico critica y evalúa los resultados de un problema o de un estudio realizado por otros (Tauber, 2010).

\subsection{Modelo de Gal (2004)}

Considerando las descripciones de los procesos cognitivos mencionados, el modelo teórico de enseñanza sobre la alfabetización estadística que plantea Gal (2004) vincula estos procesos en dos componentes: un componente de conocimiento que involucra una alfabetización estadística (formado por cinco elementos cognitivos: habilidades de alfabetización, conocimiento estadístico, conocimiento matemático, contexto de conocimiento y habilidades críticas) y un componente disposicional dado por la postura crítica, creencias y actitudes. Afirma además, que estos componentes no se pueden considerar independientes, sino como un conjunto dinámico de conocimientos y aptitudes que forman el comportamiento «estadísticamente alfabetizado», puesto que la valoración crítica de la información estadística, luego de ser comprendida e interpretada, depende de la habilidades para realizar preguntas y para impulsar una postura crítica, las cuales se basan en determinadas creencias y actitudes.

\subsection{Herramientas metodológicas del Enfoque Ontosemiótico de la Cognición y la Instrucción Matemática (EOS)}

Para realizar el análisis de las producciones de los estudiantes se utilizan herramientas metodológicas del Enfoque Ontosemiótico de la Cognición y la Instrucción Matemática (EOS), desarrollado por Godino, Batanero y Font (2009). Este marco teórico considera práctica matemática a cualquier acción, expresión o manifestación (lingüística o de otro tipo) realizada por alguien para resolver problemas matemáticos, comunicar la solución obtenida a otras personas, validar y generalizar esa solución a otros contextos. El significado de un objeto matemático, es definido como el sistema de prácticas operativas y discursivas para resolver un cierto tipo de problemas asociados a ese objeto (Godino, Batanero y Font, 2009). Ante un determinado objeto matemático, cada alumno le asigna un significado personal a este objeto. El significado fijado por el profesor, o por el libro de texto, es su significado institucional. A partir de esta distinción se puede describir el aprendizaje como acoplamiento progresivo entre significados personales e institucionales. Debido al rol preponderante que juegan los objetos matemáticos, el EOS considera que el problema epistémico-cognitivo no puede desligarse del ontológico. Así, los tipos de objetos primarios, están constituidos por:

- Situaciones-problemas: aplicaciones extra-matemáticas, ejercicios.

- Elementos lingüísticos: términos, expresiones, notaciones, gráficos, en diversos registros (escrito, oral, etc.)

- Conceptos-definiciones: introducidos mediante definiciones o descripciones.

- Proposiciones o propiedades: enunciados sobre conceptos.

- Procedimientos: algoritmos, operaciones, técnicas de cálculo.

- Argumentos: enunciados usados para validar o explicar las proposiciones y procedimientos (deductivos o de otro tipo).

Las seis entidades primarias no son objetos aislados sino que se vinculan entre sí: las situaciones-problemas son el origen y motivación de la actividad, el lenguaje actúa como soporte para representar a las restantes entidades y sirve de instrumento para la acción, los argumentos justifican los procedimientos y las proposiciones que, conjuntamente con las definiciones, resuelven las situaciones-problemas. Estas relaciones entre los objetos primarios determinan las configuraciones (Figura 1), definidas por Godino, Batanero y Font (2009) como «las redes de objetos intervinientes y emergentes de los sistemas 
de prácticas y las relaciones que se establecen entre los mismos» (p. 8). En los casos en que estas redes se refieren a acciones representativas de la institución y acordes a ella, se denominan configuraciones epistémicas. Paralelamente, las configuraciones cognitivas, son aquellas que describen los siste- mas de prácticas personales. Tanto los sistemas de prácticas como las configuraciones (epistémicas y cognitivas) se proponen como herramientas teóricas para describir los conocimientos matemáticos, en su doble versión, institucional y personal. (Godino, Contreras y Font, 2006)

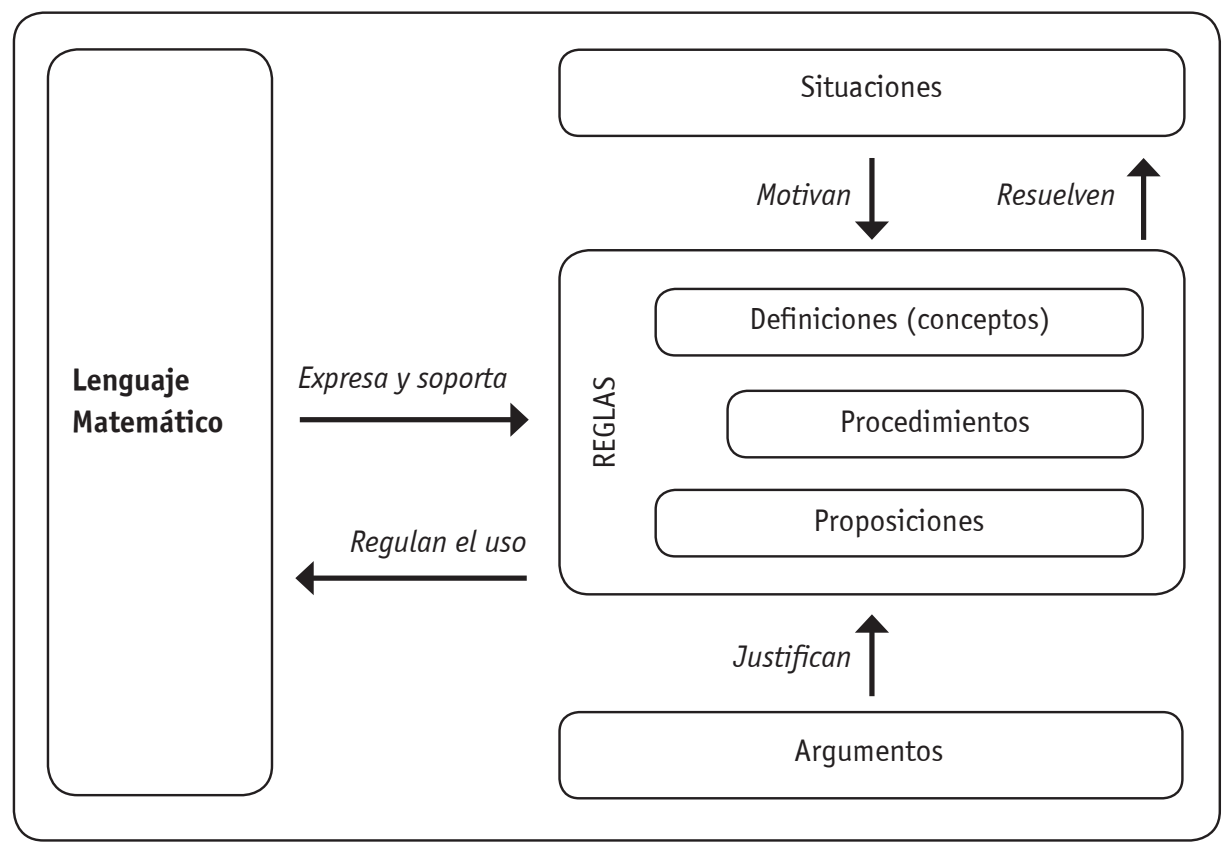

Figura 2.

Componentes de una configuración epistémica/ cognitiva. (Font y Godino, 2006).

\section{Metodología}

El trabajo identifica las prácticas matemáticas de los estudiantes con algunas de las habilidades definidas como componentes del razonamiento estadístico, según el modelo de Gal, y se detallan en los apartados siguientes.

Asimismo, dentro de las formas fundamentales de razonamiento estadístico que plantean Wild y Pfannkuch (1999), el trabajo también investiga la transnumeración, es decir, la comprensión de los estudiantes en el cambio de representación de los datos para la resolución del problema planteado. Características de los participantes: La mayoría de los estudiantes de ingeniería tiene 20 años, ya que la asignatura pertenece al 2 do año de la carrera y es cuatrimestral, con una cantidad aproximada entre 140 y 160 estudiantes por cuatrimestre, repartidos en dos comisiones. Para aumentar su participación en clase, se recurre a estrategias como el aula invertida. El trabajar en grupos ayuda en la comunicación entre pares y docentes, junto a dos informes de problemas integradores que los estudiantes suben, por grupos, al aula virtual.

Resultado de Aprendizaje del que se desprenden los criterios de evaluación de la unidad de Estadística Descriptiva.

«Utiliza la Estadística para la resolución de proble- 
mas de la ingeniería como un instrumento de resolución de problemas de análisis de datos, aplicando métodos y técnicas estadísticas para una variable».

Criterios de evaluación (CE) comprendidos:

CE1: Aplicar las etapas del método estadístico en la resolución de problemas de análisis de datos.

CE2: Calcular e interpretar el significado de las medidas de tendencia central y de posición en el contexto de la variable estadística, según la información presentada.

CE3: Analizar variabilidad y simetría en una distribución de datos.

CE4: Construir, leer e interpretar los distintos tipos de gráficos según los tipos de variables.

CE5: Comparar distribuciones de datos relacionando medidas de tendencia central y de variabilidad en forma gráfica y analítica.

Condiciones en que se llevó a cabo la enseñanza de la unidad de Estadística Descriptiva

Durante la primera clase, se plantea una actividad de Resolución de problemas de análisis de datos, donde a partir de la simulación de mediciones con recursos informáticos, se compara el calibrado de dos máquinas para la toma posterior de decisiones. Este problema disparador se va enriqueciendo con los saberes de las unidades siguientes, integrando conceptos y procedimientos hasta la comparación de medias mediante pruebas de hipótesis. Los estudiantes trabajan en grupos, definen y clasifican las variables del problema para graficar según el tipo de variable. A las variables cuantitativas continuas, las agrupan por intervalos, (siempre que se trabaje con muestras de al menos 20 datos) y luego las representan utilizando histogramas o polígonos de frecuencias. El profesor muestra cómo utilizar el software Geogebra en clase para que los estudiantes lo puedan utilizar en sus informes y trabajos.

Para la segunda clase, se aplica la estrategia del aula invertida, para ello es importante que los estudiantes hayan asimilado previamente lo trabajado durante la clase anterior y la presentación de la clase con los contenidos de Estadística Descriptiva en el aula virtual, referido ahora a las medidas de variabilidad. También calculan e interpretan medidas de tendencia central y las relacionan para analizar la simetría de la distribución. En esta segunda clase, los estudiantes resuelven problemas para el cálculo e interpretación de medidas de posición, y la detección de valores atípicos en el gráfico de caja y bigotes. Calculan e interpretan medidas de variabilidad para responder las preguntas del problema y resuelven actividades que relacionan medidas de tendencia central y de variabilidad en forma analítica y gráfica. Posteriormente, algunos grupos exponen los resultados obtenidos en una puesta en común. Como una de las actividades a evaluar, surge el problema presentado en este trabajo, cuyo objetivo consiste que el estudiante relacione la media y la desviación estándar y las vincule con el tipo de gráfico que corresponde a esas medidas, analizando la simetría de las distribuciones y la dispersión de los datos. El problema fue adaptado de unos ejercicios sobre la vinculación de estas medidas, con gráficos que no marcaron frecuencias en el eje Y. Esta forma incompleta de presentación de los datos y sin contexto en este caso, tuvo la intención de que el análisis se centre en el Criterio de Evaluación 5, (CE5): Comparar distribuciones de datos relacionando medidas de tendencia central y de variabilidad en forma gráfica y analítica, para evaluar habilidades referidas a relacionar medidas de tendencia central y de variabilidad y vincularlas con los gráficos presentados, sin tener que efectuar cálculos utilizando frecuencias.

Se espera que para su resolución, el estudiante decida, a partir de los parámetros dados, a qué poblaciones corresponden a través de su interpretación gráfica, aplicando sus conocimientos de análisis de forma y de variabilidad de los datos.

El estudio de las resoluciones de los estudiantes, es efectuada a través del análisis de los significados personales que resulta de la comparación explicitada en el criterio de evaluación 5 (CE5). Para ello, se construyó una configuración epistémica del problema, es decir, se describieron los objetos primarios intervinientes: situación problema, lenguaje, definiciones, propiedades, procedimientos 
y argumentos. Luego del análisis, se clasificaron las resoluciones y se estudiaron las dificultades encontradas, determinando en qué porcentaje se manifestaron las habilidades estadísticas evaluadas.

\subsection{Configuración epistémica}

La descripción de la configuración epistémica comienza en el apartado siguiente con el problema presentado a los estudiantes.

\subsubsection{Situación problema}

En la tabla 1 se muestran las medias y las desviaciones estándar de cuatro poblaciones. Sus gráficas se presentan en la figura 3 NO respectivamente. Identificar cada población con su gráfica correspondiente. Justificar cada elección.

\begin{tabular}{|l|r|c|c|c|}
\hline Población & \multicolumn{1}{|c|}{ A } & B & C & D \\
\hline $\begin{array}{l}\text { Media } \\
\text { Desviación } \\
\text { estándar }\end{array}$ & 198,5 & 198,1 & 193 & 193,4 \\
\hline
\end{tabular}

Tabla 1.

Medias y desviaciones estándar de cuatro poblaciones.

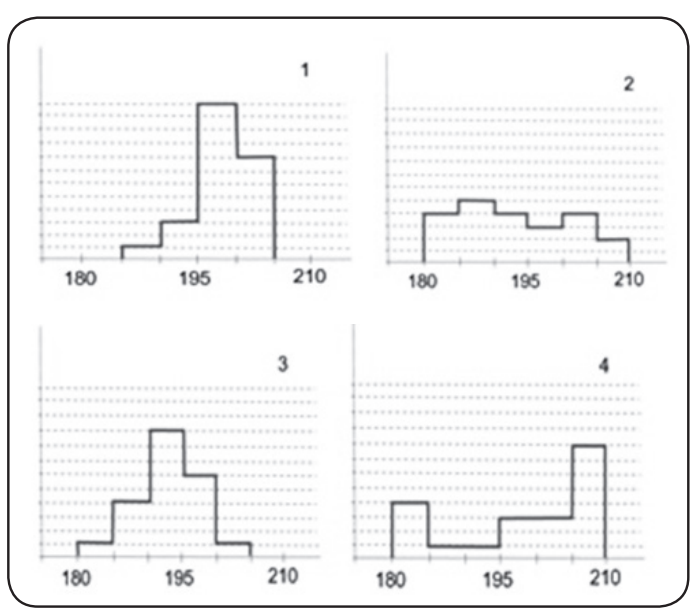

Figura 3.

Gráficas de cuatro poblaciones.
La asociación correcta en respuesta al problema, se muestra en la Tabla 2.

\begin{tabular}{|l|r|r|r|r|}
\hline Población & A & B & C & D \\
\hline Media & 198,5 & 198,1 & 193 & 193,4 \\
$\begin{array}{l}\text { Desviación } \\
\text { estándar }\end{array}$ & 9,7 & 3,9 & 4,6 & 8,08 \\
No de Gráfico & 4 & 1 & 3 & 2 \\
\hline
\end{tabular}

Tabla 2.

Asociación correcta en respuesta al problema.

\subsubsection{Procedimientos y Argumentos}

Procedimiento y Argumento 1

Comparación de las poblaciones por la aproximación de sus medias. La Argumentación 1, desarrollada en la Solución 1.

\section{Solución 1}

Al comparar las poblaciones A y B, cuyas medias se encuentran alrededor de 198 , se descartan las distribuciones de frecuencias de los gráficos 2 y 3 , porque el peso de sus frecuencias indica que sus medias respectivas están por debajo del valor 195, que es menor a 198.

Respecto de las desviaciones, se compara la desviación de la distribución de frecuencias del gráfico 4 , que resulta ser mayor a la desviación de la distribución de frecuencias del gráfico 2. Por lo tanto, se asocia el gráfico 4 con la población A y el gráfico 1 con la población $B$, tal como lo indican los datos del cuadro del enunciado del problema.

Para las poblaciones $C$ y $D$, que tienen medias similares, quedan las distribuciones de frecuencias de los gráficos 2 y 3 . Comparando nuevamente las desviaciones estándar, el gráfico 2 corresponde a la población $D$, ya que su desviación es mayor y se observa claramente en el gráfico, mientras que el gráfico 3 corresponde a la población C, donde puede observarse una menor desviación estándar en una distribución aproximadamente simétrica de los datos. 


\section{Procedimiento y Argumento 2}

Comparación de las poblaciones por el estudio de la variabilidad a través de los gráficos y / o del cálculo del coeficiente de variabilidad de Pearson. La Argumentación 2, desarrollada en la Solución 2.

\section{Solución 2}

Si se comienza por analizar la variabilidad en forma gráfica, de acuerdo al rango de la variable y a la distribución de frecuencias, cuanto más «aplanado» sea el histograma, mayor variabilidad habrá en los datos. Se eligen las poblaciones A y D por su mayor variabilidad. En consecuencia, se analizan las distribuciones de frecuencias de los gráficos 2 y 4 que muestran mayor variabilidad.

Si se analiza el gráfico 4, la media de los datos debiera ser mayor a 195 por el peso de sus frecuencias. La población A cumple con esa condición: su media es 198,5 le corresponde el gráfico 4.

Luego la distribución de frecuencias del gráfico 2 corresponde a la población $\mathrm{D}$, porque también puede observarse una media menor de 195 en su distribución de frecuencias.

Si se analiza la variabilidad en forma analítica, teniendo en cuenta que cada media es distinta, se efectúa la comparación de las poblaciones por el estudio de la variabilidad a través de los gráficos y / 0 del cálculo del coeficiente de variabilidad de Pearson:
$\mathrm{C}_{\mathrm{VA} \%}=9,7 / 198,5 \%=0,048.100=4,8 \%$;

$\mathrm{C}_{\mathrm{vB} \%}=3,9 / 198,1 \%=0,020.100=2 \%$

$C_{\mathrm{vc} \%}=4,6 / 193 \%=0,024.100=2,4 \%$;

$C_{\mathrm{vD} \%}=8,08 / 193,4 \%=0,042 \cdot 100=4,2 \%$.

De acuerdo a los porcentajes obtenidos, se ubica cada uno de los valores dados de las medias, en los gráficos respectivos, para la asociación de la gráfica con sus parámetros.

\section{Procedimiento y Argumento 3}

Este procedimiento recurre al cambio de representación de los datos. A pesar de que se excluyeran frecuencias en los gráficos para que no fueran utilizadas, a los estudiantes les alcanzó esta lectura incompleta de los gráficos, para asignar frecuencias a cada intervalo, utilizando la escala del eje «y», marcada sólo con líneas punteadas. La Argumentación 3, en la Solución 3.

\section{Solución 3}

Los estudiantes utilizaron la escala del eje «y» del gráfico. Con ella, determinaron frecuencias para cada intervalo del gráfico respectivo, como se muestra en la tabla 3, y luego calcularon la media y la desviación estándar, a partir de la información deducida, para compararlas con los parámetros dados.

\begin{tabular}{|l|r|l|r|l|l|l|l|}
\hline Gráfico 1 & Fi & Gráfico 2 & fi & Gráfico 3 & fi & Gráfico 4 & fi \\
\hline$[180-185)$ & 0 & {$[180-185)$} & 4 & {$[180-185)$} & 1 & {$[180-185)$} & 4 \\
{$[185-190)$} & 1 & {$[185-190)$} & 5 & {$[185-190)$} & 4 & {$[185-190)$} & 1 \\
{$[190-195)$} & 3 & {$[190-195)$} & 4 & {$[190-195)$} & 9 & {$[190-195)$} & 1 \\
{$[195-200)$} & 10 & {$[195-200)$} & 3 & {$[195-200)$} & 6 & {$[195-200)$} & 3 \\
{$[200,205)$} & 6 & {$[200,205)$} & 4 & {$[200,205)$} & 1 & {$[200,205)$} & 3 \\
{$[205,210)$} & 0 & {$[205,210)$} & 2 & {$[205,210)$} & 0 & {$[205,210)$} & 8 \\
Totales & 20 & Totales & 22 & Totales & 20 & Totales & 20 \\
\hline
\end{tabular}

Gráfico 1: Media 198,1 y Desviación Estándar 3,9; corresponde a la Población B. Gráfico 2: Media 193,4 y Desviación Estándar 8,08; corresponde a la Población D. Gráfico 3: Media 193 y Desviación Estándar 4,6; corresponde a la Población C. Gráfico 4: Media 198,5 y Desviación Estándar 9,7; corresponde a la Población A.
Tabla 3.

Tabla de frecuencias a partir de la escala del eje «y» con la determinación de frecuencias posibles entre los datos de los gráficos. 


\subsubsection{Lenguaje}

Lenguaje Simbólico

Media poblacional:

$\mu$ Desviación Estándar poblacional: $\sigma$

Coeficiente poblacional de variabilidad porcentual: $\mathrm{C}_{\mathrm{v} \%}=\frac{\sigma}{\mu} \cdot 100$

Media muestral:

$\bar{x}$ Desviación Estándar muestral: $\mathrm{S}$

Coeficiente muestral de variabilidad porcentual: $\mathrm{C}_{\mathrm{v} \%}=\frac{S}{\bar{x}} \cdot 100$

\section{Lenguaje Gráfico}

Para variables cuantitativas discretas: gráfico de bastones (con frecuencias absolutas, relativas 0 porcentajes).

Para variables cuantitativas continuas: Histogramas o polígonos de frecuencias (con frecuencias absolutas, relativas o porcentajes). Para intervalos regulares, cada caso es sólo un cambio de escala. Otra forma de representación de los datos es a través de la representación tabular de los mismos, donde se utilizan frecuencias absolutas, relativas o porcentajes. Para intervalos regulares, cada caso es sólo un cambio de escala.

\subsubsection{Definiciones}

Media Poblacional: es el promedio aritmético de todos los valores de la variable $X$ de una población. Desviación Estándar poblacional: es la raíz cuadrada del promedio del cuadrado de los desvíos.

Coeficiente de variabilidad porcentual: es el producto entre el cociente de la desviación estándar y la media, con el número 100.

Mediana (Me), es el valor central del conjunto ordenado de datos.

Moda (Mo), es el valor de la variable $X$ asociado a la mayor frecuencia.

Rango $\left(R=X_{\text {máx }}-X_{\text {min }}\right)$, es la diferencia entre el valor máximo y el valor mínimo de la variable.

\subsubsection{Propiedades}

Relaciones entre la media, la mediana y la moda y la forma de la distribución de los datos:

Si la distribución es simétrica, la media, mediana y moda son aproximadamente iguales. El coeficiente de asimetría es próximo a cero.

Si la distribución asimétrica es positiva, el coeficiente de asimetría es positivo.

Si la distribución es asimétrica negativa, el coeficiente de asimetría es negativo.

Si el coeficiente de variabilidad porcentual es menor al $30 \%$, se asume poca variabilidad en los datos.

\subsection{Habilidades consideradas para el análisis del pensamiento estadístico según el modelo de Gal (2004)}

La componente de conocimiento, se codificó de esta forma: $\mathrm{H}_{1}$ : habilidades de alfabetización, $\mathrm{H}_{2}$ : conocimiento estadístico.

A las habilidades de alfabetización estadística $\left(\mathrm{H}_{1}\right)$ se las desagrega según la definición dada en la introducción respecto de la alfabetización estadística, referida a la lectura e interpretación básica de la información y de los resultados: $\mathrm{H}_{11}$ : Organizar datos, construir y presentar tablas y trabajar con distintas representaciones de datos. $\mathrm{H}_{12}$ : Comprensión básica de conceptos, vocabulario y símbolos.

El conocimiento estadístico $\left(\mathrm{H}_{2}\right)$ está dado por el razonamiento estadístico, es decir, por cómo razonar y dar sentido a la información estadística. En esta habilidad no fue considerado el pensamiento estadístico, ya que no se pretende formar habilidades de estadísticos profesionales, sino formar en competencias de la asignatura, como se expresó en un apartado del marco teórico. En consecuencia, al desagregar el conocimiento estadístico $\left(\mathrm{H}_{2}\right)$, se estudiaron $\mathrm{H}_{21}$ : Realizar interpretaciones basadas en un conjunto de datos, representar o resumir datos, $\mathrm{H}_{22}$ : Efectuar relaciones entre conceptos, $\mathrm{y}_{23}$ : Interpretar, de manera global, los resultados estadísticos. Tal como indica Gal, el resto de las habilidades de la componente de conocimiento, es decir, el conocimiento matemático, el contexto de conocimiento y 
las habilidades críticas, no son independientes de las anteriores, se consideraron implícitas en las habilidades codificadas, porque hubo casos en que, para la resolución del problema, se efectuaron cálculos matemáticos, se aplicó el razonamiento estadístico y se tomaron decisiones a partir de ciertos criterios. En cuanto a las creencias y actitudes de los estudiantes, que corresponden a la componente disposicional, no fueron contempladas en este trabajo. En el apartado siguiente, se relacionan las habilidades consideradas del modelo y las prácticas matemáticas evaluadas.

\subsection{Habilidades de Alfabetización y de Conocimiento Estadístico desagregadas y su vinculación con los objetos primarios evaluados en la configuración epistémica}

Con la intención de expresar las habilidades en términos de las prácticas matemáticas evaluadas, y posteriormente, establecer una correspondencia con los tipos de respuestas de los estudiantes a la problemática planteada, se muestra esta vinculación en la Tabla 4.

\begin{tabular}{|l|l|l|}
\hline $\begin{array}{l}\text { Habilidades consideradas } \\
\text { del Modelo de Gal (2004) }\end{array}$ & Desagregado de las Habilidades & $\begin{array}{l}\text { Objetos primarios evaluados } \\
\text { en la configuración epistémica }\end{array}$ \\
\hline $\begin{array}{l}\mathrm{H}_{1} \text { : Habilidades de } \\
\text { alfabetización }\end{array}$ & $\begin{array}{l}\mathrm{H}_{11}: \text { Organizar datos, construir y presentar } \\
\text { tablas y trabajar con distintas representacio- } \\
\text { nes de datos. } \\
\mathrm{H}_{12}: \text { Comprensión básica de conceptos, } \\
\text { vocabulario y símbolos. }\end{array}$ & $\begin{array}{l}\text { Solución 3 } \\
\text { Procedimiento y Argumento } 3\end{array}$ \\
\hline $\begin{array}{l}\mathrm{H}_{2}: \text { Habilidades de } \\
\text { conocimiento estadístico }\end{array}$ & $\begin{array}{l}\mathrm{H}_{21}: \text { Realizar interpretaciones basadas en un } \\
\text { conjunto de datos, representar o resumir datos. } \\
\mathrm{H}_{22}: \text { Efectuar relaciones entre conceptos. } \\
\mathrm{H}_{23}: \text { Interpretar, de manera global, los resul- } \\
\text { tados estadísticos. }\end{array}$ & $\begin{array}{l}\text { Soluciones } 1 \text { ó 2 Procedimiento } \\
\text { Argumento 1 ó 2 }\end{array}$ \\
\hline
\end{tabular}

Tabla 4.

Habilidades de Alfabetización y de Conocimiento Estadístico y su vinculación con las Objetos primarios evaluados en la Configuración Epistémica. 


\section{Resultados}

La categorización del pensamiento estadístico a través de las habilidades consideradas, se muestran en la Tabla 5 con los tipos de resoluciones obtenidas. El análisis se efectuó con los estudiantes que resolvieron el problema dado que, para las categori- zaciones así definidas, la ausencia de respuesta no aporta información en el estudio.

Para poder visualizar y comparar los tipos de resoluciones, se muestra la figura 4.

\begin{tabular}{|c|c|c|c|c|c|c|c|c|c|}
\hline \multirow{2}{*}{$\begin{array}{l}\text { Habilidades } \\
\text { consideradas } \\
\text { por Gal (2004) }\end{array}$} & \multirow{2}{*}{$\begin{array}{l}\text { Objetos primarios } \\
\text { evaluados en la } \\
\text { configuración } \\
\text { epistémica }\end{array}$} & \multicolumn{8}{|c|}{$\begin{array}{l}\text { Porcentaje de estudiantes que evidenciaron } \\
\text { estas habilidades según su resolución R } \\
\text { (RC: Correcta, RPC: Parcialmente Correcta, RI: Incorrecta) }\end{array}$} \\
\hline & & $\mathrm{RC}$ & $\begin{array}{l}\text { RC } \% \\
18 \%\end{array}$ & RPC & $\begin{array}{r}\text { RPC \% } \\
\quad 3 \%\end{array}$ & RI & $\begin{array}{l}\text { RI \% } \\
0.9 \%\end{array}$ & $\begin{array}{r}\text { Totales } \\
24\end{array}$ & $\begin{array}{r}\text { Totales } \% \\
21 \%\end{array}$ \\
\hline $\begin{array}{l}\mathrm{H}_{2}: \\
\text { Habilidades de } \\
\text { conocimiento } \\
\text { estadístico }\end{array}$ & $\begin{array}{l}\text { Procedimiento } 1 \\
\text { y Argumento } 1 \\
\text { ó } \\
\text { Procedimiento } 2 \\
\text { y Argumento } 2\end{array}$ & 32 & $28 \%$ & 11 & $9 \%$ & 46 & $41.1 \%$ & 89 & $79 \%$ \\
\hline Totales & & 52 & $46 \%$ & 14 & $12 \%$ & 47 & $42 \%$ & 113 & $100 \%$ \\
\hline
\end{tabular}

Tabla 5.

Clasificación de los tipos de resoluciones de los estudiantes según las habilidades de alfabetización estadística evaluadas del modelo de enseñanza de Gal (2004).

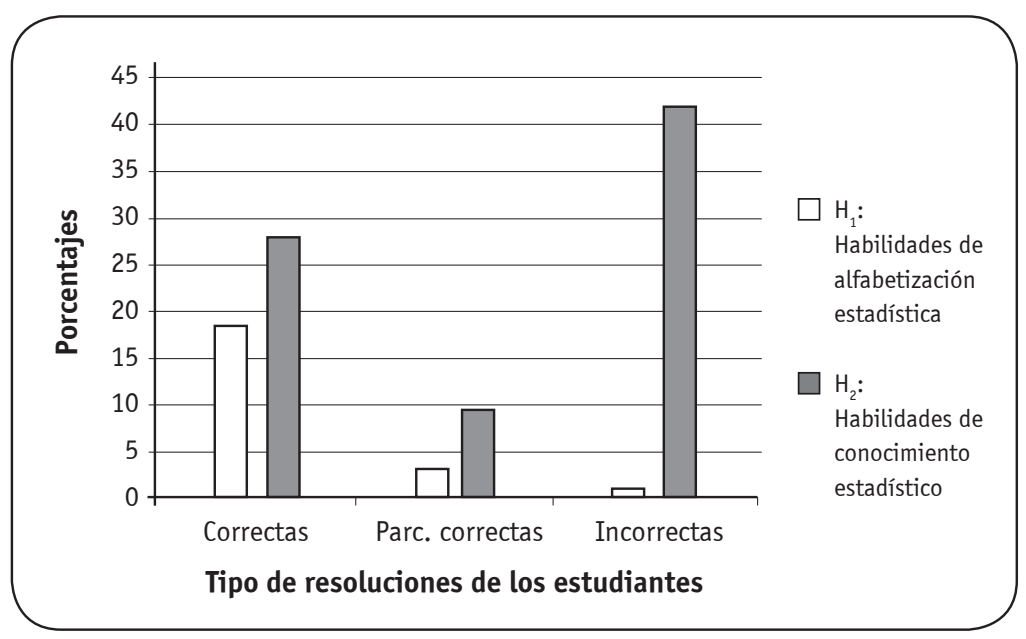

Figura 4.

Clasificación de los tipos de resoluciones de los estudiantes según las habilidades evaluadas. 


\subsection{Análisis de los resultados}

Una lectura del Gráfico1 muestra que casi el 80\% de los estudiantes, decidió realizar un análisis cualitativo del problema, es decir, aplicaron los conocimientos estadísticos que vinculan los gráficos con los parámetros, resumidos en las habilidades caracterizadas por $\mathrm{H}_{2}$, Habilidades de Conocimiento Estadístico. El resto, abordó el problema utilizando la transnumeración, es decir, cambió la forma de representación de los datos para la resolución del problema, utilizando Habilidades de Alfabetización Estadística, codificadas como $\mathrm{H}_{1}$.

Un $40 \%$ de las resoluciones con herramientas asociadas a $\mathrm{H}_{2}$ (los conocimientos estadísticos que vinculan los gráficos con los parámetros), resultaron incorrectas.

Un mejor resultado fue observado en las producciones en las que se aplicó la transnumeración, ya que en casi todas, resolvieron correctamente el problema. El problema fue planteado con la intención de evaluar los significados asociados a Habilidades de Conocimiento Estadístico $\left(\mathrm{H}_{2}\right)$, y a pesar de que la mayor parte de los estudiantes pretendió abordar el problema aplicando estas prácticas matemáticas, en aproximadamente la mitad de las producciones, se evidenciaron errores tanto en procedimientos como en argumentaciones. Sólo un $26 \%$ de estudiantes vinculó correctamente los parámetros dados con los gráficos y efectuó un análisis coherente respecto de la ubicación de la media y la forma de las distribuciones. Estas prácticas matemáticas están descriptas en la configuración epistémica con los procedimientos 102 , justificados con sus respectivas argumentaciones.

A continuación se describen dos casos particulares que deben atenderse.
En la Figura 5 se muestra la respuesta de un estudiante en las que se observan algunos conflictos de significados. Calcula el coeficiente de variación tomando la media y la desviación como valores muestrales, en lugar de tomarlos como parámetros de las poblaciones dadas. Esta confusión en el uso de símbolos, es un conflicto semiótico potencial que debe resolverse antes de introducir los temas de inferencia, manifiesta que el estudiante no contextualiza adecuadamente la información dada, al confundir la notación de la media y el desvío estándar de una población, con la media y el desvío estándar de una muestra. Posteriormente, analiza la variabilidad de los datos, averiguando que si el coeficiente de variación porcentual es menor que el $30 \%$, se ocupa de estudiar la representatividad de la media de los datos, olvidando que la representatividad de la media es válida en distribuciones simétricas.

En la Figura 6, se expone una resolución en la que se registraron otras dificultades. Además del conflicto de significados al simbolizar el coeficiente de variación, en sus argumentaciones se evidencia que no tiene construido el concepto de simetría, ya que por ejemplo, asocia el gráfico 3 (Ver Figura 3) a la población $\mathrm{D}$ que, por sus parámetros, no lo es.

Un caso interesante de resolución correcta se presenta en la Figura 7, en la que se observa una forma de resolución mixta con argumentaciones asociadas tanto a Habilidades de Alfabetización Estadística $\left(\mathrm{H}_{1}\right)$ como a Habilidades de conocimiento estadístico $\left(\mathrm{H}_{2}\right)$. La construcción de las tablas con los cálculos efectuados, evidencia la manifestación de las habilidades codificadas con $\mathrm{H}_{1}$. Las argumentaciones dadas con posterioridad, son coherentes con los cálculos efectuados y revelan las habilidades codificadas en $\mathrm{H}_{2}$. 

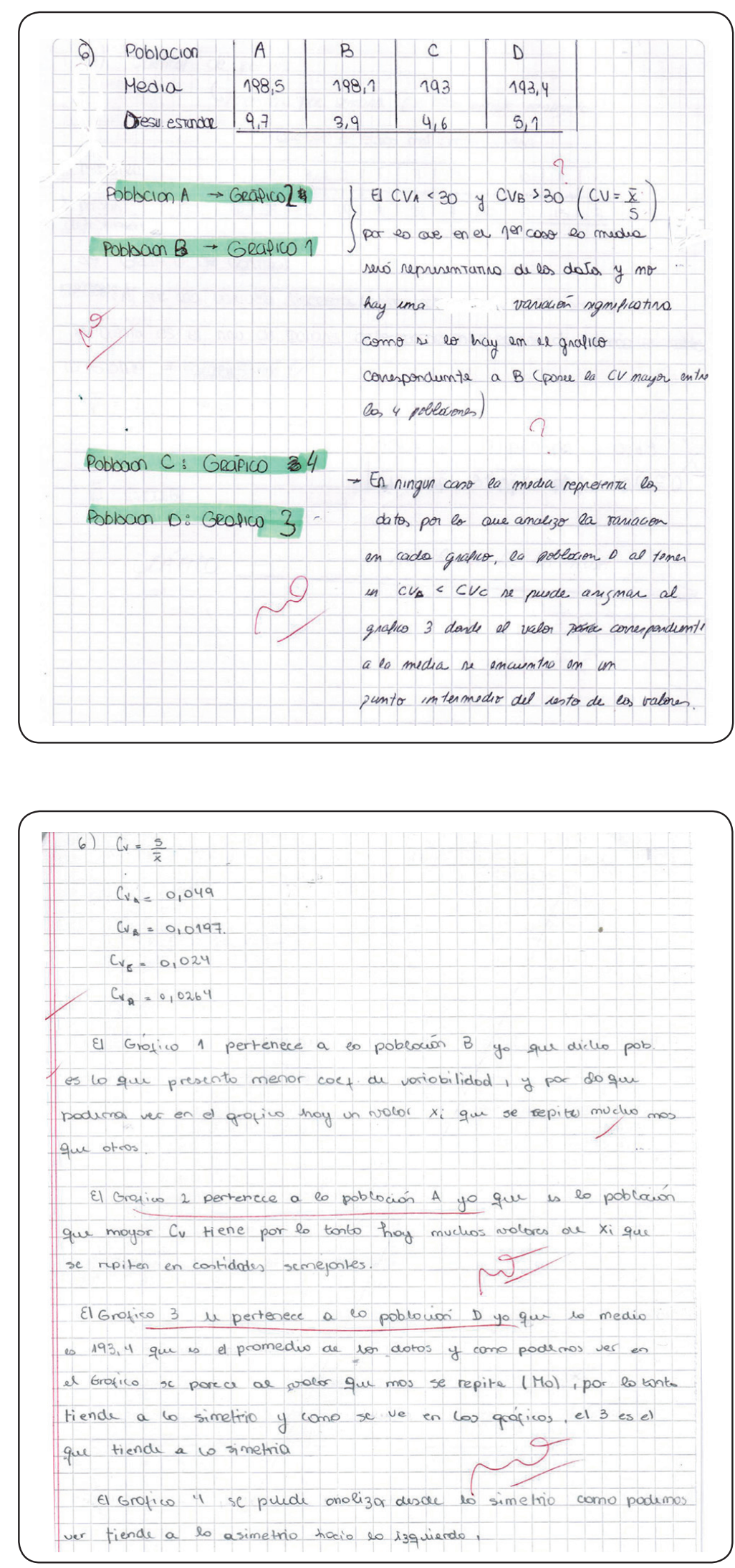

Figura 5.

Resolución en la que se observan conflictos de significados de parámetros poblacionales.
Figura 6.

Resolución en la que se observan conflictos de significados en el análisis de la simetría y variabilidad. 


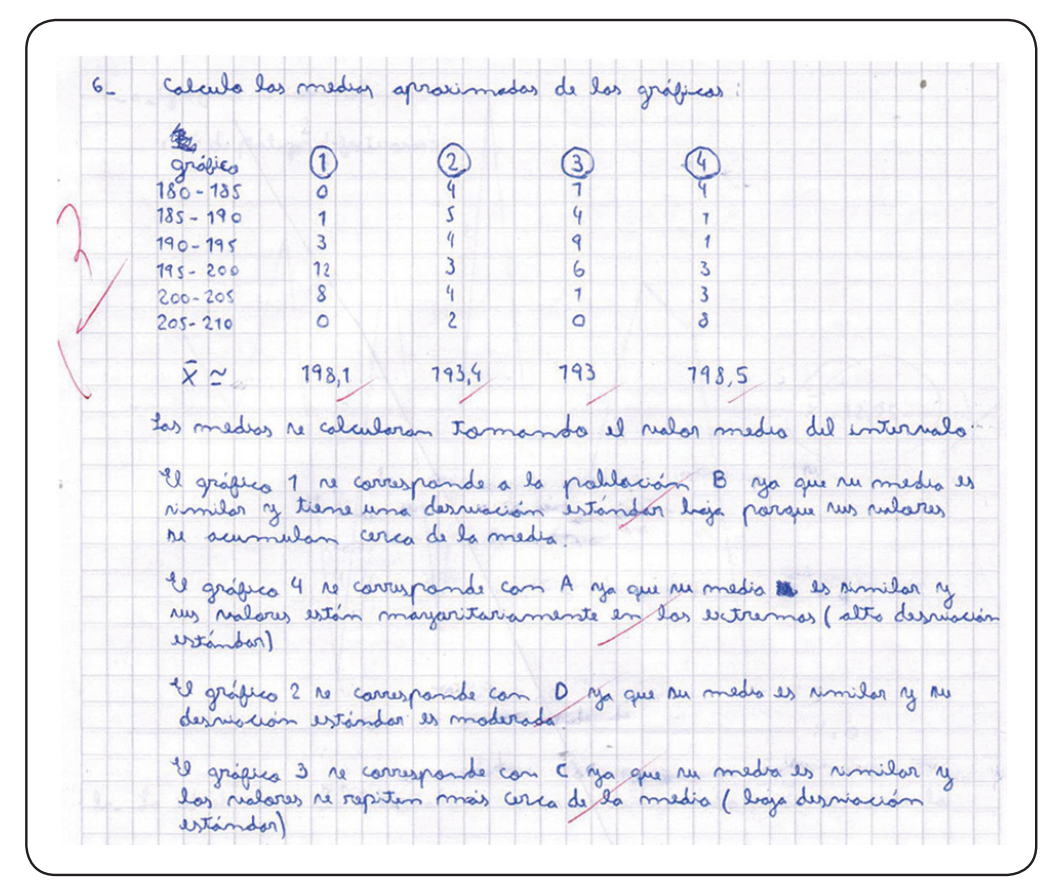

Figura 7.

Resolución en la que se observan la manifestación de $\mathrm{H}_{1}$ y $\mathrm{H}_{2}$.

\section{Conclusiones}

En este trabajo se presentó un análisis de los significados personales de parámetros vinculados a sus representaciones gráficas. Se analizaron las producciones de estudiantes de ingeniería según las habilidades detectadas en la resolución de un problema donde se evaluaron habilidades de alfabetización y de conocimiento estadístico. Cada una de ellas fue desagregada en habilidades vinculadas a los procedimientos aplicados en los diferentes tipos de resolución del problema planteado.

Del análisis de las producciones, se comprobó que las Habilidades del Conocimiento Estadístico no están afianzadas en la mayor parte de los estudiantes. Pero se encontraron habilidades no esperadas y perfectamente afianzadas en la resolución del problema. Es el caso de los estudiantes que recurrieron al cambio de representación de los datos. A pesar de la omisión de frecuencias en los gráficos para que no fueran utilizadas, fue suficiente para los estudiantes la lectura incompleta de los mismos para asignar frecuencias a cada intervalo, utilizando la escala del eje «y», marcada sólo con líneas punteadas. El hecho que los intervalos de los gráficos sean regulares, hizo que las frecuencias sean proporcionales a la escala considerada y pudieran resolver el problema haciendo un cambio del registro gráfico al tabular, es decir aplicando la transnumeración.

Este procedimiento es un elemento a tener en cuenta en el planteo de este tipo de problemas, como docentes investigadores aportó conocimiento didáctico del contenido estadístico. Surge preguntarse entonces cómo hubieran respondido los estudiantes si los intervalos hubieran sido irregulares. Esta cuestión abre posibilidades en el planteo de problemas cuyo abordaje didáctico favorezca la construcción de significados acerca del razonamiento estadístico que considere el cambio de registros, como una habilidad de transnumeración que dará lugar a variantes en la presentación de problemáticas asociadas al criterio evaluado y por qué no a los otros criterios mencionados en este trabajo.

En ese sentido, no puede dejar de mencionarse que las situaciones problemas a plantear en clase, deban abarcar a todos estos criterios de evaluación mencionados porque entre todos ellos se evalúa el razonamiento estadístico en el contexto de la ingeniería. 


\section{Referencias bibliográficas}

Arteaga, P., Batanero, C., Díaz, C. y Contreras, J.M. (2009). El lenguaje de los gráficos estadísticos. Unión, 18, 93-104. Madrid. FISEM. Consultado el 3/12/2019 en https://dialnet.unirioja.es/servlet/ articulo?codigo $=5900401$

Batanero, C. (2013). Sentido Estadístico: Componentes y desarrollo. I Jornadas virtuales en Didáctica de la Estadística, Probabilidad y Combinatoria. Consultado el 3/12/2019 en http://www.ugr. es/ $\sim$ batanero/pages/ARTICULOS/Sentidoestad\%C3\%ADstico.pdf

Batanero, C.; Godino, J.; Green, D.; Holmes, P.; Vallecillos, A. (1994). Errors and difficulties in understanding statistical concepts. International Journal of Mathematics Education in Science and Technology, 25(4), 527-547.

Ben-Zvi, D.; Garfield, J. (2004). Statistical Literacy, Reasoning and Thinking: goals, definitions and challenges. D. Ben-Zvi y J. Garfield (eds.), The challenge of developing statistical literacy, reasoning and thinking, 3-15

CONFEDI (2007). Competencias Genéricas. Desarrollo de competencias en la enseñanza de la ingeniería argentina. San Juan. Universidad Nacional de San Juan.

CONFEDI (2018). Propuesta de estándares de segunda generación para la Acreditación de Carreras de Ingeniería. Disponible en https://confedi.org.ar/librorojo/

Curcio, F. (1989). Developing graph comprehension. National Council of Teachers of Mathematics, Reston, VA.

Figueroa, S. y Baccelli, S. (2018). Mejora de una propuesta didáctica sobre la vinculación entre una variable estadística y su variable aleatoria asociada. Actas de la XXXI Reunión Latinoamericana de Matemática Educativa (ALME 31), 31 (1), 257-264.

Font, V. y Godino, J. (2006). La noción de configuración epistémica como herramienta de análisis de textos matemáticos: su uso en la formación de profesores. Educaçao Matemática Pesquisa, 8 (1), 67-98. Gal, I. (2004). Statistical Literacy: meanings, components, responsibilities. En: D. Ben-Zvi y J. Garfield (eds.) The challenge of developing statistical literacy, reasoning and thinking, 47-78

Godino, J.; Contreras, A.; Font, V. (2006). Análisis de procesos de instrucción basado en el enfoque ontológico semiótico de la cognición matemática. Recherches en Didactique des Mathématiques, 26(1), 39-88

Godino, J.; Batanero, C.; Font, V. (2009). Un enfoque ontosemiótico del conocimiento y la instrucción matemática. Versión ampliada del artículo The onto-semiotic approach to research in mathematics education. ZDM. The International Journal on Mathematics Education, 39(1-2) 127-135 Consultado el 20 de mayo de 2018 en http://die.udistrital.edu.co/sites/default/files/doctorado_ud/publicaciones/ parte_i_un_enfoque_ontosemiotico_del_conocimiento_y_instruccion_matematica.pdf

Tauber, L. (2010). Análisis de elementos básicos de alfabetización estadística en tareas de interpretación de gráficos y tablas descriptivas. Ciencias Económicas. Investigación 8(1), 53-74.

Wild, C.; Pfannkuch, M. (1999). Statistical thinking in empirical enquiry. International Statistical Review. 67(3), 221-248 